\title{
Disrupters as Well as Monitors: Roles of Others During and After Collaborative Remembering in the DRM Procedure
}

\author{
Aiqing Nie, Chunchun Ke, Mengsi Li, and Bingyan Guo \\ Department of Psychology and Behavioral Science, Zhejiang University, Hangzhou 310028, China
}

ABSTRACT

\section{KEYWORDS}

collaborative inhibition

error pruning

source retrieval

post-collaborative recall DRM

\begin{abstract}
Influence of others on true and inauthentic memory both during and after collaborative remembering have drawn extensive attention in recent years. Collaborative research has recorded three typical effects: collaborative inhibition and error pruning (i.e., nominal groups recall more true information but also bear higher erroneous intrusions than collaborative groups) during collaboration, as well as post-collaborative recall benefit after collaboration. This study introduced DeeseRoediger-McDermott (DRM) lists not only to investigate these phenomena in semantically related information, but also set a course to explore false memory in the collaborative context. Another issue is the sensitivity of these effects to different episodic memory tests (i.e., item memory and source memory tests). In views of these, the current study instructed participants to study several DRM lists and then recall previously studied words (item recall) together with their displayed colors (source retrieval) twice (Recall 1 and 2). Recall 1 was performed either individually or collaboratively, whereas Recall 2 was conducted individually. The cost of collaborative inhibition was obtained, along with three different beneficial effects: error pruning, false memory reduction, and post-collaborative recall benefit. Furthermore, the novel implication of the current study is that it reveals the sensitivity of collaborative inhibition and error pruning in DRM lists to testing conditions and demonstrates that the modulation of collaboration on false memory occurs in the same way both during and after collaboration. These results are discussed in terms of the retrieval strategy disruption hypothesis and other accounts.
\end{abstract}

\section{INTRODUCTION}

\section{Collaborative Memory and Its Paradigm}

In daily life, we are accustomed to creating and retrieving shared events with other people. For example, a group of college students may gather together to recall the content of a lecture in cognitive psychology before the final exam. Scenarios like this involving two or more people remembering together are termed the collaborative memory (Basden, Basden, \& Henry, 2000; Marion \& Thorley, 2016; Meade \& Roediger, 2009; Rajaram \& Pereira-Pasarin, 2010; Weldon \& Bellinger, 1997). The dominant approach to investigate collaborative memory in laboratory settings involves participants studying a list of items on their own and subsequently performing a free recall test: some complete the recall task individually while others work together (Barber, Castrellon, Opitz, \& Mather, 2017; Barber, Rajaram, \& Aron, 2010; Marion \& Thorley, 2016; Sjolund, Erdman, \& Kelly, 2014; Wessel, Zandstra, Hengeveld, \& Moulds, 2015).

It has been confirmed in this way that memory performance is superior when people recall collaboratively versus individually (Barber et al., 2017; Sjolund et al., 2014; Wessel et al., 2015). However, in contrast Corresponding author: Aiqing Nie, Department of Psychology and Behavioral Science, Zhejiang University, 148 Tianmushan Road, Hangzhou 310028, China. E-mail: nieaiq@126.com 
with such an intuitive mnemonic boost caused by adding contributors, what concerns researchers more is the extent to which collaborative members are capable of achieving their potentials during collaboration. To evaluate this, researchers compare the performance of a collaborative group with that of a virtual one termed the nominal group (Blumen, Rajaram, \& Henkel, 2013; Hyman, Cardwell, \& Roy, 2013; Rajaram \& Pereira-Pasarin, 2010). A collaborative group is composed of two (dyads), three (triads), or occasionally four (tetrads) members performing a memory task together. As for a nominal group, it should equal the collaborative group in size and it is formed by randomly assigning the members or creating all possible groupings of individual participants. The nominal group's performance is the pooling of the nonredundant items recalled. For example, if one person in a dyad recalls the items $A, B$, and $C$, and the other recalls the items $A, C$, and $D$, then the nonredundant recalling performance of this nominal group contains $A, B, C$, and D (Barber et al., 2017; Marion \& Thorley, 2016; Wessel et al., 2015). Studies following this data analysis strategy have obtained significantly weaker performance for collaborative groups versus nominal groups (called collaborative inhibition), which confirms collaborative remembering to be a detrimental process that prevents group members from recalling up to their full potentials. Nevertheless, there is also a beneficial side, given that strikingly fewer errors have been observed for collaborative groups than nominal groups (called error pruning, Barber, Rajaram, \& Fox, 2012; Harris, Barnier, \& Sutton, 2012; Hyman et al., 2013; Sjolund et al., 2014; Weldon, Blair, \& Huebsch, 2000; Wessel et al., 2015).

\section{Possible Explanations for Costs and Benefits of Collaborative Remembering}

Regarding the costs and benefits of collaborative remembering, there are several probable social and cognitive explanations. Karau and Williams (1993) explain the collaborative inhibition as the production of social loafing, whereby group members are reluctant to put forth their greatest efforts due to the responsibility diffusion. Complementally, Andrews and Rapp (2015) regard collaborative inhibition as the result of weaker motivation in collaborative groups and error pruning as the product of participants' fear of negative evaluation from others for making mistakes, so that much stricter response criteria are applied. On the other hand, a widely accepted cognitive interpretation is the retrieval strategy disruption hypothesis (RSDH) proposed by Basden, Basden, Bryner, and Thomas (1997). The RSDH claims that each individual possesses a preexisting cognitive structure, by which an idiosyncratic cognitive organization for studied items is developed. However, a participant's idiosyncratic cognitive organization and their preferred retrieval strategies are easily disrupted by the presence of others, resulting in collaborative inhibition (Barber et al., 2012; Barber et al., 2017; Barber, Harris, \& Rajaram, 2015; Basden, Basden, \& Stephens, 2002; Congleton \& Rajaram, 2014; Garrido, Garcia-Marques, \& Hamilton, 2012; Hyman et al., 2013; Marion \& Thorley, 2016; Rajaram, 2011; Rajaram \& Pereira-Pasarin, 2010; Weldon et al., 2000). Furthermore, this hypothesis ascribes error pruning to collaborative members' monitoring of answers, feedback, and curtailing of errors for each other (Rajaram, 2011)

\section{False Memory in the Collaborative Memory Paradigm Using Deese- Roediger-McDermott Stimuli}

Collaborative inhibition and error pruning demonstrate inverse roles of others in collaborative item recall: disadvantageous disrupters in true memory but benign monitors and/or correctors in inauthentic memory. Many studies have contributed to understanding the mechanism of collaborative inhibition and revealed multiple factors. For instance, it was stronger in a collaborative group with a bigger size (Basden et al., 2000; Thorley \& Dewhurst, 2007), much higher in emotional versus neutral words (Ke, Nie, \& Zhang, 2017), weaker for acquainted partners than strangers (Harris, Barnier, Sutton, \& Keil, 2014; Harris, Barnier, Sutton, Keil, \& Dixon, 2017), and was reduced by repeated learning (Basden et al., 2000; Blumen \& Rajaram, 2008). Comparatively, the error pruning effect has been relatively overlooked, with the most notable results concerning the collaborating style: error pruning occurred when collaborators were allowed to communicate in a free-flowing manner and to reach a consensus; no such phenomenon was produced in a turn-taking procedure where participants were required to wait until their turns to retrieve, prohibiting talk and corrections or comments on one another's outputs (Harris et al., 2012; Rajaram, 2011).

As mentioned above, prior studies not only understated error pruning to some extent, but also failed to control the generation of memory errors. One way to make up for these deficiencies is to adopt the Deese-Roediger-McDermott (DRM) lists as stimuli in the collaborative memory paradigm. In a DRM procedure, the to-be-remembered words (e.g., winter, snow, and shiver) in a list are all semantically related to an unstudied critical word (e.g., cold, Numbers, Meade, \& Perga, 2014; Roediger \& McDermott, 1995; Thorley \& Dewhurst, 2007). A typical finding is that the unstudied critical word is frequently falsely remembered as studied, and such an error is termed false memory (Numbers et al., 2014). Thus, it allows for exploration on the potential influence of collaborative remembering on this experimentally induced memory illusion and for probe into the collaborative inhibition in mnemonic information with inherent semantic relevance.

Existing research concerning collaborative remembering of DRM lists is inconclusive. Most studies applying the free-flowing method identified the standard collaborative inhibition as well as diminished false memory for collaborative versus nominal groups (Maki, Weingold, Arrelano, 2008; Takahashi, 2007; Weigold, Russell, \& Natera, 2014), with only one study that found neither of these effects (Thorley \& Dewhurst, 2007). However, Thorley and Dewhurst reported evident collaborative inhibition and higher false recall of critical lures in collaborative groups under the turn-taking condition. A recent study following the turn-taking procedure (Saraiva, Albuquerque, \& Arantes, 2017) also found the collaborative inhibition but lower false memories for collaborative versus nominal groups. Considering the disparate results between Thorley and Dewhurst and other studies, we 
hoped to provide further evidence for collaborative inhibition and the beneficial effect of false memory reduction in DRM lists using a freeflowing procedure.

Meanwhile, we also kept an eye on falsely recalled noncritical words to address the standard error pruning, which has been largely ignored in the extant literature. The rationale behind this lies in the potential diverging generation mechanisms between critical and noncritical items. Critical lures are experimentally invited through the activation of semantic relations. As to the wrongly outputted noncritical words, their generation is spontaneous and their causes could be more diversified. Aside from the semantic relation that gives rise to the critical lures, perceptual characteristics (e.g., phonological association, glyph association, etc.) of the to-be-remembered items might also contribute to error intrusions (Qu \& Ding, 2010). Thorley and Dewhurst (2007) also addressed falsely outputted noncritical items, but failed to confirm significant error pruning in either the free-flowing or the turn-taking conditions. Their results suggested more noncritical errors for collaborative groups versus the nominal groups in the turn-taking condition. Such a data pattern is inconsistent with error pruning recorded in semantically unrelated stimuli and thus deserves further investigation.

\section{Collaborative Remembering in Source Memory}

Episodic memory is the memory of self-experienced events that can be explicitly stated. Previous research has identified two subtypes of episodic memory: Item memory that retrieves the core item information (e.g., item recall) and source memory that retrieves the detailed contextual information associated with the items (e.g., source retrieval, Barredo, Öztekin, \& Badre, 2015; Bell, Mieth, \& Buchner, 2017; Cooper, Greve, \& Henson, 2017; Leynes, Crawford, Radebaugh, \& Taranto, 2013; Mitchell \& Johnson, 2009; Nie, Guo, Liang, \& Shen, 2013; Nie, Jiang, Fu, \& Zhang, 2015). Compared with the extensive concern of collaborative memory research in item recall mentioned above, collaborative remembering literature concerning source memory is scarce (Andersson, 2001; Ke et al., 2017; Sjolund et al., 2014; Wessel et al., 2015). However, source memory bears higher ecological relevance compared to item memory since real-life scenes not only contain simple items, but are rich in contextual details. For instance, when driving with a friend to find a restaurant that you both have once been to, you might work together to recollect its location.

Existing research, though rare, has consistently established evident collaborative inhibition at source retrieval. For instance, both Andersson (2001) and Sjolund et al. (2014) found worse performance in spatial source retrieval for collaborative versus nominal groups. Wessel et al. (2015) obtained a reliable effect of collaborative inhibition at retrieval of source information in an emotional film, and significant error pruning as well. Our recent study (Ke et al., 2017) compared collaborative inhibition and error pruning between item memory and color source memory. We found much higher collaborative inhibition and much weaker error pruning on item recall than source retrieval. Considering that stimuli applied in the former task were without semantic relation, the current study's second aim was to examine whether similar data patterns could be replicated using DRM lists.

The different sensitivities of item recall and source retrieval to collaboration might be explained through the following logic. The dual-process memory theory has defined two processes underpinning memory retrieval: the automatic familiarity process accompanied by a sense of knowing (which has usually been linked to item recognition), as well as the deliberate recollection process with autonoetic recovery of encoding episodes (Bell et al., 2017; Cooper et al., 2017; Dulas \& Duarte, 2013; Malejka \& Bröder, 2016; Mollison \& Curran, 2012; Wixted, 2007; Yonelinas, Aly, Wang, \& Koen, 2010). Both item recall and source retrieval are supposed to rely on recollection in a substantial degree (Hayama, Vilberg, \& Rugg, 2012; Kragel \& Polyn, 2016; Rajan \& Bell, 2015). Nevertheless, evidence also suggests that item recall with successful source retrieval would recruit recollection to a higher extent. Support could be drawn from the fact that item recall accompanied by successful rather than unsuccessful source retrieval was associated with enhanced activity in cortical regions involved in the recollection network (including bilateral parietal, posterior midline, and parahippocampal cortex, Hayama et al., 2012). Accordingly, we assumed that source retrieval relied on the controlled recollection process to a greater extent in contrast with item recall. Based on this assumption and the RSDH, the collaborative inhibition in source retrieval should exceed that in item recall, since a more controlled memory type should allow resistance to retrieval disruptions from collaborative members in group remembering

\section{Post-Collaborative Recall Benefit and the Account}

Collaboration can not only affect memory in an ongoing way, but also have a lasting impact. Several experiments set two or more successive recall tests instead of one to examine whether prior collaborative experience impacts later individual memory (Bärthel, Wessel, Huntjens, \& Verwoerd, 2017; Basden et al., 2000; Blumen \& Rajaram, 2008; Blumen, Young, \& Rajaram, 2014; Congleton \& Rajaram, 2011; Harris et al., 2012). After their first recall for studied items (Recall 1), all participants are informed to make subsequent recall(s) on their own (e.g., Recall 2). Accordingly, during Recall 2, the participants from previous individual and collaborative groups are labeled as II (Individual-Individual) and CI (Collaborative-Individual) groups, respectively. Studies on such later individual memory revealed the post-collaborative recall benefit. It manifests in higher performance for participants who have engaged in individual recall after collaboration than those who have not collaborated before (i.e., CI performed better versus II), and suggests that collaboration could promote later individual memory (Bärthel et al., 2017; Basden et al., 2000; Blumen \& Rajaram, 2008; Congleton \& Rajaram, 2011; Harris et al., 2012). Furthermore, repeated collaboration elicited greater post-collaborative recall benefit than a single-time one (Blumen et al., 2014; Blumen \& Stern, 2011).

The RSDH proposes several mechanisms in which collaboration can be beneficial for later individual memory. For the first point, compared with nominal members, collaborative members are able to gain 
re-exposure to events recalled by their partners during the previous retrieval, which leads to improvements in their later retrieval. The second is the relearning through retrieval: collaboration can produce powerful effects on relearning by enabling rehearsal of studied information. The third explanation is error-pruning-interaction with collaborative partners and receiving their feedback helps prune the errors one might otherwise make in individual recall (Blumen et al., 2014; Blumen \& Rajaram, 2008; Harris et al., 2012; Marion \& Thorley, 2016; Rajaram \& Pereira-Pasarin, 2010; Wissman \& Rawson, 2015). However, it is still unclear whether the post-collaborative recall benefit could be observed when applying DRM lists. This was the third aim of our study.

\section{The Current Study}

In summary, the current study aimed to present a comprehensive depiction of collaborative remembering in DRM stimuli by investigating the following aspects: (a) whether significant collaborative inhibition, error pruning, and post-collaborative recall benefit could be recorded; (b) whether the two episodic memory tests (i.e., item recall and source retrieval) could yield similar patterns of the above-mentioned three effects; and (c) whether false memory differed between collaborative and nominal groups. Notably, false memory refers specifically to falsely outputted critical lures during retrieval, whereas when calculating the amount of error pruning, critical lures were excluded and only falsely retrieved noncritical words were contained. In this sense, an experiment with three phases (i.e., study, Recall 1, and Recall 2) was conducted. We adopted DRM lists as materials and assigned the displayed colors of words as sources. During the study phase, words were displayed in different colors. Both recalls included item recall (i.e., to recall studied words) and source retrieval (i.e., to retrieve previously displayed color for each word) testing conditions.

During Recall 1, the performance of nominal groups was compared with that of collaborative groups regarding the recall of presented words together with their colors. We expected collaborative inhibition and error pruning in both item recall and source retrieval. Particularly, we anticipated much stronger collaborative inhibition and much weaker error pruning on item recall versus source retrieval basing on our recent data pattern (Ke et al., 2017), which could verify a deeper dependence of source retrieval versus item recall on the controlled recollection process. Furthermore, considering the production of false memories for critical lures, we predicted lower false recall for collaborative than nominal groups, consistent with previous studies (Maki et al., 2008; Saraiva et al., 2017; Takahashi, 2007; Weigold et al., 2014). During Recall 2, we anticipated a pronounced post-collaborative recall benefit for both memory testing conditions, as a variety of studies obtained similar benefits after collaboration (Bärthel et al., 2017; Basden et al., 2000; Blumen \& Rajaram, 2008; Harris et al., 2012). With regard to errors, we expected a larger error pruning effect and less false memory following collaboration, drawing support from the explanation offered by the RSDH (Blumen et al., 2014; Blumen \& Rajaram, 2008; Harris et al., 2012; Marion \& Thorley, 2016; Rajaram \& Pereira-Pasarin, 2010; Wissman \& Rawson, 2015).

\section{METHOD}

\section{Participants}

A total of sixty right-handed undergraduate college students, aged 18-23 years, were recruited. All were native Chinese speakers with normal or corrected-to-normal visual acuity. None reported any history of neurological or psychiatric problems or color blindness. Forty participants constituted twenty dyads for collaborative groups, with each person belonging to only one group. Twenty dyads were formed as nominal groups via pairwise matching members from the remaining participants who finished the memory tests individually; each was paired with no more than two of the other participants. All participants gave informed consent in writing and received course credit for volunteering. The Research Ethics Committee of Zhejiang University approved all protocols. At the end of the experiment, the participants were thanked and debriefed.

To evaluate whether the samples size in the current study was appropriate, we conducted a sensitivity power analysis with $\mathrm{G}^{\star}$ Power software v3.1 (Faul, Erdfelder, Buchner, \& Lang, 2009). It detected a small-to-medium-sized effect $(f=0.21)$ of the interaction between group (nominal and collaborative) and testing condition (item recall and source retrieval) using a repeated-measures analysis of variance (ANOVA) under the standard criteria ( $\alpha=0.05$ two-tailed, $1-\beta=0.80$ ), which demonstrated that our sample size met the requirement.

\section{Design}

This experiment was a $2 \times 2 \times 2$ (Group [nominal, collaborative] $\times$ Test Phase [Recall 1, Recall 2] $\times$ Testing Condition [item recall, source retrieval]) mixed design. Group was a between-subject variable, while the other two were within-subject variables. Regarding the two test phases, Recall 1 was performed either individually or collaboratively, while Recall 2 was always carried out individually.

\section{Materials}

The DRM lists were taken from the previous studies (Roediger \& McDermott, 1995; Thorley \& Dewhurst, 2007), and were translated into Chinese. According to the previous research on collaborative memory (Hyman et al., 2013; Ke et al., 2017; Wissman \& Rawson, 2015), seventytwo words in total, that is, six twelve-word DRM lists were chosen as stimuli in our formal experiment (see the Appendix for the English versions). The words in each list were all semantically related to one critical word, which would not be presented during the study phase. To prevent interference across different lists, the whole experiment was divided into six blocks, namely, one list per block. The order of the lists was pseudorandomized. Twelve words of an additional seventh list severed as practice materials. To note, the backward associative strength (BAS) of items, which measures the associations strength for words in a DRM list to the critical lure, was neither controlled nor analyzed in the current experiment, since the stimuli were borrowed from different studies (Roediger \& McDermott, 1995; Thorley \& Dewhurst, 2007) and not all of them had provided the BAS. 


\section{Procedure}

To ensure a thorough understanding of the instructions, all participants went through practice trials before the formal experiment. All protocols were identical between the practice and the formal experiment, including one study phase and two test phases (i.e., Recall 1 and Recall 2) in each block, both of which had the testing conditions of item recall together with source retrieval.

\section{STUDY PHASE}

During the study phase of each block, participants were separately seated in front of a monitor $60 \mathrm{~cm}$ away and their sight was at the same height as the screen center. Each trial began with the presentation of a fixation cross in the center of the monitor for $500 \mathrm{~ms}$. Then, a word was presented for $1 \mathrm{~s}$, which was followed by a $1 \mathrm{~s}$ inter-stimulus interval. The words were displayed one at a time and were pseudo-randomly presented. Half of the words were displayed in red and the other half in green. For each block, the colors of the words were randomly distributed. Participants were instructed to memorize each word and discriminate the displayed colors by pressing two different keys: $F$ (or $J$ ) for red words, $J$ (or $F$ ) for green words. Keys were pressed with the index fingers, while the assignments of the fingers were counterbalanced among blocks for each participant. Participants were encouraged to respond as quickly and accurately as possible. Participants in individual groups and the dyads for subsequent collaboration all studied alone, seated in front of separate monitors at separate tables. The dyads for collaboration were informed to start each block simultaneously. After the study and tests of a whole block, a five-minute resting interval was provided before the sequential block to relieve the participants' fatigue.

All the to-be-remembered words were presented in Arial font. Words were programmed by E-prime software v3.0 (Psychology Software Tools, INC) and were centrally displayed against a black background on a Sony CRT monitor. Figure 1 illustrates the study procedure with some word samples, in which the trials of 冬天 (winter), 下雪 (means snow), and 发抖 (means shiver) are all semantically related to a nonpresented critical word cold.

\section{TEST SESSIONS}

Test sessions. The testing conditions were set by a sequential paradigm —one of the widely utilized paradigms to investigate source memory, in which two responses were performed consecutively to an item: retrieving the item and then the encoding context associated with it (Cycowicz \& Friedman, 2003; Nie et al., 2013; Nie, Guo, \& Shen, 2011; Ventura-Bort et al., 2016; Yick \& Wilding, 2014). Members of individual groups retrieved the studied words on their own twice, whereas participants of collaborative groups retrieved the words first in a group context, and then individually. All recalls were performed via a paper-and-pencil pattern in a free-recall manner. Since Recall 1 and Recall 2 were distinct from each other, they are described separately.

\section{RECALL 1}

At Recall 1 of each block, participants recalled information either individually (nominal recall) or collaboratively (collaborative recall). Participants in the nominal condition were instructed to recall on their own as many studied words as they could in a free order, as well as the previously displayed color for each recalled word. Recalled words and the associated colors were written down on a prepared sheet within a time limit of three minutes. The collaborative dyads performed the task in a free-flowing procedure-both members tried to retrieve as many items as they could in no particular order (Marion \& Thorley, 2016). They were instructed to discuss the task together, with one of the two members responsible for writing down the answers within the limit of three minutes. Neither remembering turns nor other manipulations were emphasized to collaborative dyads.

\section{RESTING INTERVAL}

When Recall 1 of each block was finished, all participants were given a resting interval of two minutes before Recall 2 .

\section{RECALL 2}

During Recall 2, all participants were informed to recall each studied word as well as its previously displayed color separately and wrote down

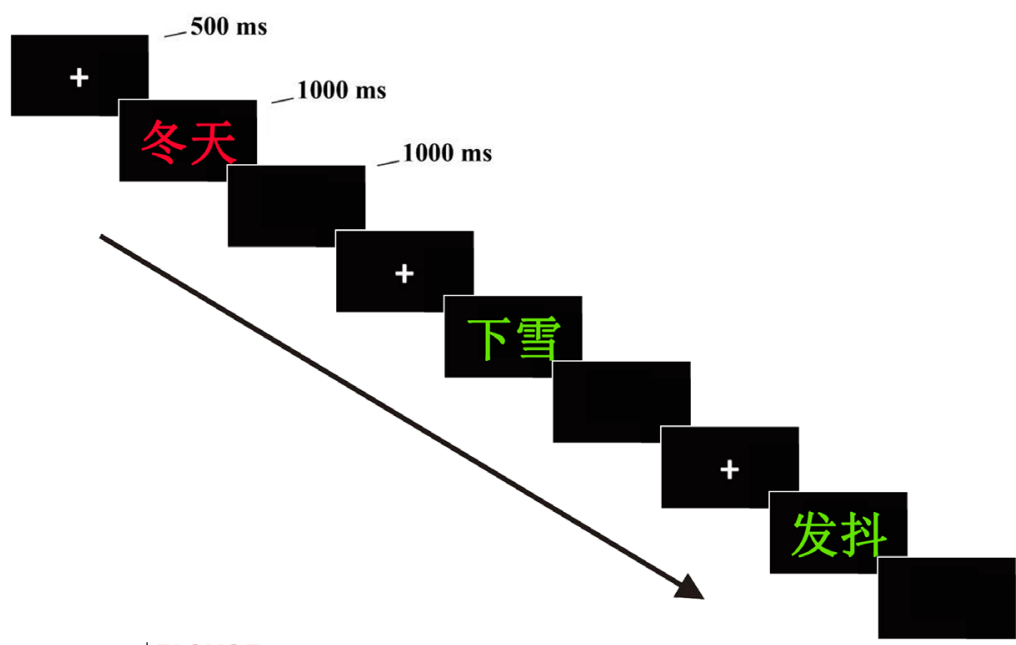

FIGURE 1.

Schematic illustration of the study phases and word samples. 
the answers on a prepared sheet within three minutes. All participants were told to avoid the interference caused by Recall 1 as much as they could. The purpose was to lower the potential intrusions of the erroneously retrieved information from Recall 1.

\section{RESULTS}

During the study, the color discrimination performance was above $95 \%$ for all participants. Hence, only the performance during retrieval was concerned. For both Recall 1 and 2, data were analyzed with IBM SPSS Statistics v22 (IBM Corporation, 2014). All inferential analyses used an a level of 0.05 (two-tailed). We mainly reported the reliable results pertinent to the current study's aims.

\section{Data Analyses and Results of Recall 1}

During Recall 1, as in previous literature (Barber et al., 2017; Ke et al., 2017; Marion \& Thorley, 2016; Wessel et al., 2015), the redundant information recalled were removed to form the nominal group recall. Furthermore, due to the paper-and-pencil format, only the answers written down were analyzed; the ones that were discussed but not written down were not considered. Data analyzed in the current study mainly focused on the raw recalling numbers instead of on proportions. We did so to ensure the consistency of data analyses, since the erroneous recalling data could not be presented in the probabilistic pattern. However, to provide a comprehensive understanding of the results, the proportions of correct source identifications to the number of correct recalls were also calculated, since a sequential paradigm was applied where source memory was dependent on item memory.

For Recall 1, total numbers of both the correctly recalled words at item recall and the correctly recalled colors at source retrieval are shown in Figure 2. The proportions of correct source identifications to the number of correct recalls are shown in Table 1 . To analyze whether reliable collaborative inhibition was acquired, a $2 \times 2$ (Group [nomi-

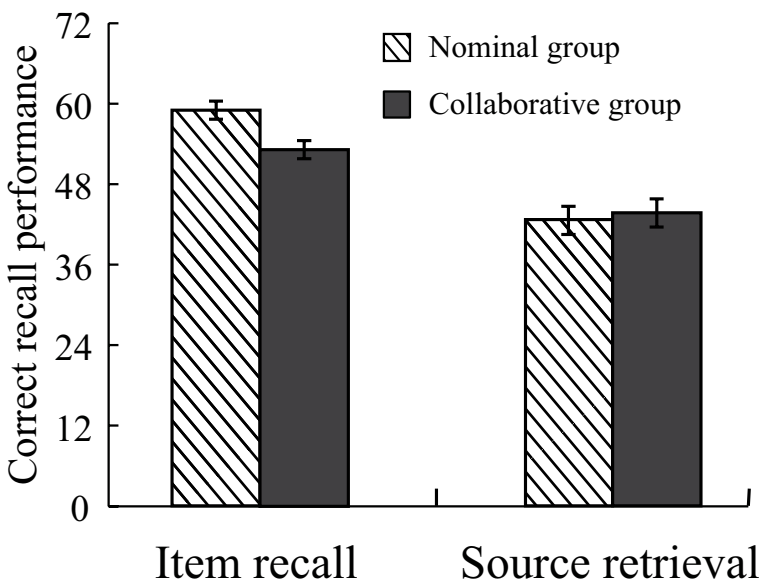

|FIGURE 2.

Total number of correctly recalled words at item recall and correctly recalled colors at source retrieval during Recall 1. nal, collaborative] $\times$ Testing condition [item recall, source retrieval]) repeated-measures ANOVA was conducted. The ANOVA did not reveal a significant main effect of group, $F(1,19)=0.917, p=.350$, $\eta^{2}=0.046$, but a significant main effect of testing condition, $F(1,19)$ $=138.123, p<.001, \eta^{2}=0.897$, as well as their interaction, $F(1,19)=$ $11.290, p=.003, \eta^{2}=0.373$. Hereafter, simple effect analyses indicated that at item recall, nominal groups yielded significantly better performance than collaborative groups, $F(1,19)=9.10, p=.007$. At source retrieval, nominal and collaborative groups showed nearly equivalent performance, $p=.729$. The significant performance difference between nominal and collaborative groups at item recall indicated significant collaborative inhibition for this testing condition during Recall 1.

Since we applied a sequential paradigm to test item memory and source memory, the source memory performance was dependent on that of item memory. Therefore, in addition to analyses conducted on the numbers of correct source retrieval, we analyzed the proportions of correct source identifications to the number of the correctly recalled items. To analyze whether reliable collaborative inhibition could be obtained, an independent-sample t-test with group (nominal, collaborative) as a variable was performed. This analysis revealed a significant effect, $t(38)=-2.546, p=.015, d=-0.805$, which indicated higher proportions of correct source identifications for collaborative versus nominal groups, and suggested no collaborative inhibition when applying proportion data, but a reverse pattern instead.

To further explore the sensitivity of collaborative inhibition to testing condition, the magnitude of collaborative inhibition (i.e., total number difference between nominal and collaborative group recall) was analyzed. An independent-samples $t$-test with testing condition (item recall, source retrieval) as the variable was performed. The analysis revealed a marginally significant effect for this factor, $t(38)=$ $1.851, p=.072, d=0.290$, showing a larger magnitude of collaborative inhibition at item recall versus source retrieval and indicating that the magnitude of this effect was sensitive to testing condition.

During Recall 1 , the total number of errors, the incorrectly recalled unstudied words at item recall, as well as the incorrectly recalled colors for both recalled studied and unstudied words at source retrieval are shown in Figure 3. The unstudied words at source retrieval were the words that were erroneously reported as studied. Here, under both testing conditions, those of the erroneous intrusions for critical lures were not taken into consideration, as relevant data would be concerned in the analysis for false memory (see the following descriptions and The Current Study section). To analyze whether we could find reliable error pruning, a $2 \times 2$ (Group [nominal, collaborative] $\times$ testing condition [item recall, source retrieval]) repeated-measures ANOVA was

TABLE 1.

Proportions of Correct Source Identifications to Correct Recalls and False Memories at Item Recall during Recall 1

\begin{tabular}{ccccc}
\hline & \multicolumn{2}{c}{$\begin{array}{c}\text { Source-correct } \\
\text { proportions }\end{array}$} & \multicolumn{2}{c}{ False memories } \\
\hline & $\begin{array}{c}\text { Nominal } \\
\text { group }\end{array}$ & $\begin{array}{c}\text { Collaborative } \\
\text { group }\end{array}$ & $\begin{array}{c}\text { Nominal } \\
\text { group }\end{array}$ & $\begin{array}{c}\text { Collaborative } \\
\text { group }\end{array}$ \\
\hline$M$ & 0.719 & 0.821 & 2.800 & 0.750 \\
$S E$ & 0.207 & 0.272 & 0.345 & 0.160 \\
\hline
\end{tabular}




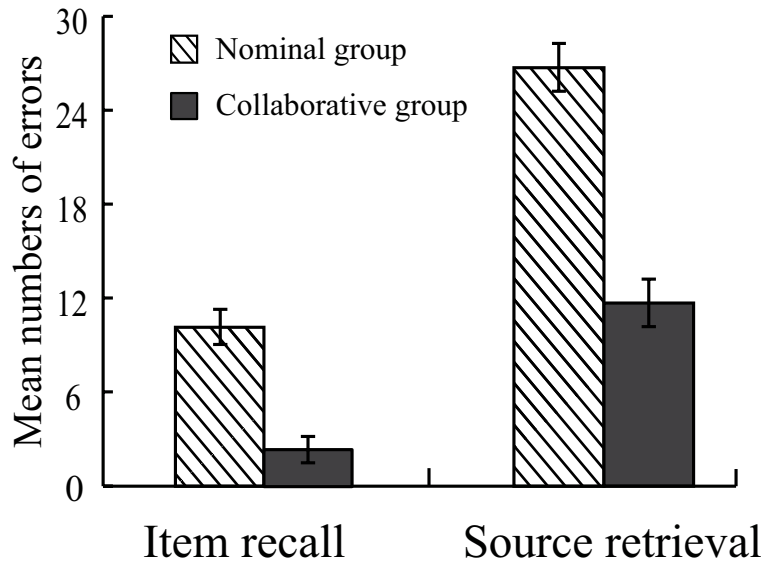

FIGURE 3.

Total number of errors (i.e., incorrectly recalled unstudied words at item recall and incorrectly recalled colors for both recalled studied and unstudied words at source retrieval) during Recall 1.

conducted. The ANOVA found reliable main effects of both variables, $F(1,19)=40.205, p<.001, \eta^{2}=0.695$, and $F(1,19)=138.123, p<$ $.001, \eta^{2}=0.879$, as well as a two-way interaction, $F(1,19)=11.290$, $p$ $=.003, \eta^{2}=0.373$. Hereafter, the simple effect analyses suggested that, at both testing conditions, the number of errors was much greater for nominal groups compared to collaborative groups, $F(1,19)=25.44$, $p$ $<.001$, and $F(1,19)=38.82, \mathrm{p}<.001$, respectively. Thus, lower errors for collaborative than nominal groups suggested that significant error pruning occurred in both testing conditions.

Specifically, to analyze the impact of the testing condition upon the amplitude of error pruning (i.e., the difference of the number of noncritical errors between nominal and collaborative groups), we performed an independent-samples $t$-test with the testing condition (item recall, source retrieval) as the variable. The analysis revealed a significant effect of testing condition, $t(38)=-2.440, p=.019, d=0.390$, demonstrating much greater error pruning on source retrieval versus item recall.

The false memories (i.e., erroneous intrusions of critical lures as studied words) at item recall during Recall 1 are shown in Table 1. As falsely reported unstudied critical lures and their displayed colors were not presented during encoding, only the performance of false memories during item recall was analyzed. To this end, an independent-samples $t$-test of group (nominal, collaborative) was conducted. The $t$-test revealed a significant effect of group, $t(38)=5.391, p<.001, d=0.85$, which indicated that more false memories were produced by nominal groups compared with collaborative groups, and in turn, suggested that collaboration could effectively reduce the erroneous intrusions of critical lures.

\section{Data Analyses and Results of Recall 2}

Analyses for Recall 2 held the same strategy. To be specific, the data analyzed mainly focused on the raw recalling numbers rather than proportional data. Since all participants completed the memory task individually during Recall 2 , they were assigned into two groups according to their collaborative history during Recall 1 . Given that nominal groups were virtual during Recall 1 , members who had been in this group were defined as II during Recall 2. In contrast, those who had performed Recall 1 in collaborative groups formed the CI group during Recall 2. Figure 4 reflects the numbers of both correctly recalled words as well as the correctly recalled colors during Recall 2 . To analyze whether we obtained a reliable post-collaborative recall benefit, a 2 $\times 2$ (Group [II, CI] $\times$ Testing Condition [item recall, source retrieval]) repeated-measures ANOVA was conducted. The ANOVA revealed a significant main effect of group, $F(1,19)=5.473, p=.025, \eta^{2}=0.126$, showing better remembering performance for CI than II. The main effect of testing condition also reached statistical significance, $F(1,19)$ $=125.046, p<.001, \eta^{2}=0.767$, with better performance on item recall versus source retrieval. There was no significant two-way interaction, $F(1,19)=0.289, p=.594, \eta^{2}=0.008$. The greater remembering performance for CI versus II indicated a reliable post-collaborative recall benefit in both testing conditions.

The proportions of correct source identifications to the number of correct recalls are shown in Table 2. Similar to that of Recall 1, analysis on the proportions of correct source identifications to the number of correct item recalls was also conducted for Recall 2. To test whether significant post-collaborative recall benefit occurred, an independentsamples t-test with group (II, CI) as the variable was performed, but did not confirm a reliable effect, $p=.903$, demonstrating no postcollaborative recall benefit from the perspective of proportion.

The numbers of errors (i.e., incorrectly recalled unstudied words at item recall, and incorrectly recalled colors for both studied and unstudied words at source retrieval) during Recall 2 are shown in Figure 5. The unstudied words at source retrieval were those words that were erroneously reported as studied ones. Similar to Recall 1 , the erroneous intrusions of critical lures under both conditions were excluded here, as the data will be analyzed below. To analyze whether significant error pruning occurred, a $2 \times 2$ (Group [II, CI) $\times$ Testing Condition [item recall, source retrieval]) repeated-measures ANOVA was conducted. The ANOVA only revealed a significant main effect of testing condition, $F(1,19)=125.046, p<.001, \eta^{2}=0.767$. This indicated more errors at source retrieval versus item recall, but the error amount did not differ statistically between the two groups.

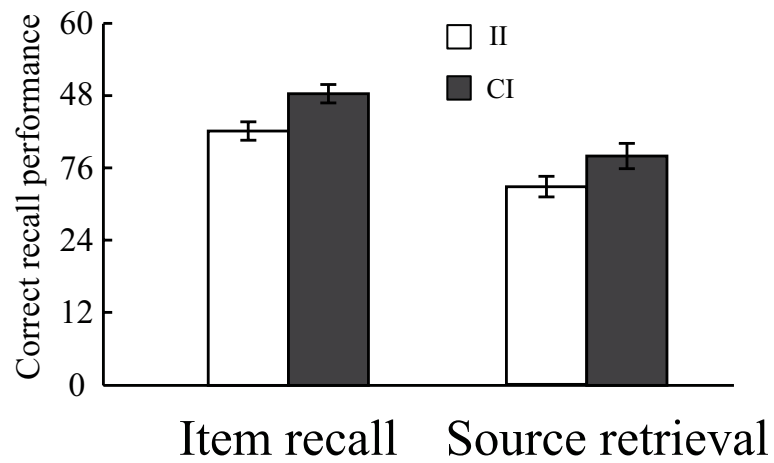

|FIGURE 4.

Total number of correctly recalled words at item recall and correctly recalled colors at source retrieval during Recall 2 . 


\begin{tabular}{lcccc}
\hline TABLE 2. & \\
Proportions of Correct Source Identifications to Correct Recalls \\
and False Memories at Item Recall during Recall 2
\end{tabular}

Note. $\mathrm{II}=$ individual-individual, $\mathrm{CI}$ = collaborative-individual.

The false memories during Recall 2 are shown in Table 2. An independent-samples $t$-test on group (II, CI) revealed a significant effect, $t(38)=2.802, p=.008, d=0.440$, with much higher false memories for II than CI groups, which suggested that prior collaboration experience could effectively reduce the erroneous intrusions of critical lures during subsequent individual recall.

\section{DISCUSSION}

The current experiment mainly focused on the costs and benefits both during and after collaboration when DRM lists were applied and two types of episodic memory tests were considered. During collaboration, group members were proved to be both detrimental disrupters and beneficial monitors, since not only the cost effect of collaborative inhibition but also the beneficial effects of error pruning and false memory reduction were observed. We also recorded the post-collaborative recall benefit and false memory reduction during the second individual recall, which confirmed that the impact of collaborative remembering could still last even after the withdraw of collaborative context. Furthermore, the sensitivity of relevant effects to testing conditions were taken into consideration innovatively.

\section{The Cost Effect of Collaborative Inhibition for Deese-Roediger- McDermott Lists is Sensitive to Testing Conditions Duuring Collaborative Remembering}

Regarding the correct responses, collaborative groups recalled items less correctly than nominal groups during Recall 1 , and this effect of collaborative inhibition confirmed a disadvantage of collaborative remembering. People were hindered from reaching their predicted potentials during collaborative recall. Such results were not only consistent with the findings of several collaborative memory studies using semantically unassociated stimuli (Barber et al., 2010; Barber et al., 2012; Barber et al., 2017; Ke et al., 2017; Sjolund et al., 2014; Wessel et al., 2015), but also replicated the studies of collaborative inhibition adopting DRM lists as stimuli (Maki et al., 2008; Takahashi, 2007; Weigold et al., 2014). Current results and previous research conjointly indicate that participants' idiosyncratic retrieval strategies vary greatly, even for stimuli with inherent semantic relations. The phenomenon can be explained through the RSDH, which states that when participants recall items together, the output of one member could disrupt the strategies of the other (Barber et al., 2012; Hyman et al., 2013; Marion \& Thorley,
2016; Rajaram, 2011; Weldon et al., 2000). This is why we defined the roles of others during collaboration as disrupters. Although applying DRM lists as materials there appeared to be no inhibition effect in one study (Takahashi, 2007), it could be explained by the fact that some of the collaborative members in that study were pairs of close friends who shared memories and knew well about each other's retrieval style, and thus similar strategies were used without disruption. Coincidentally, as was proved by another study (Harris, Barnier, \& Sutton, 2013), when group members adopt similar strategies during both encoding and retrieval phases, there is no significant collaborative inhibition.

Inconsistent with our prediction and our recent study (Ke et al., 2017), the current design did not confirm reliable collaborative inhibition on source retrieval. However, comparison of the two testing conditions did show a numerical trend of stronger collaborative inhibition for item recall versus source retrieval, which is consistent with the pattern in our recent study (Ke et al., 2017). Such a data pattern suggests that participants' retrieval strategies in source retrieval could be more convergent than in item recall, which conforms to our assumption that source retrieval might rely more deeply on the deliberate recollection process, and thus be more immune to interference. However, within the current experimental design, we were unable to measure the recollection process quantitatively. The most rigorous way to disentangle the contribution of recollection (and perhaps also familiarity) to different testing conditions would be to apply an alternative measurement model, either the process dissociation procedure (McCabe, Roediger, \& Karpicke, 2011), or the receiver operating characteristics analysis (Yonelinas \& Parks, 2007), neither of which could be conducted with the current data. Therefore, the above-mentioned speculation is merely qualitative.

The lack of collaborative inhibition at source retrieval in the current study might also be attributed to other factors. One is that direct encoding of the contexts (i.e., discriminating the colors) might facilitate subsequent source retrieval, as in our recent study (Ke et al., 2017). Thus, alternative encoding tasks are suggested in future research. Another possibility is that retrieval cues provided by a collaborator

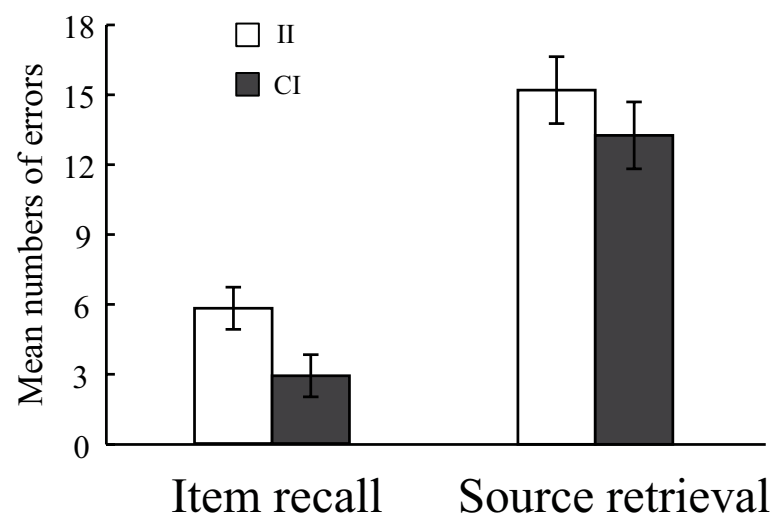

\section{FIGURE 5.}

Total number of errors (i.e., incorrectly recalled unstudied words at item recall and incorrectly recalled colors for both recalled studied and unstudied words at source retrieval) during Recall 2. 
might not have been as influential in the case of source memory as they probably were in the case of free recall, given that the source retrieval task involved only two choices. However, following this logic, memory errors should also be less affected under the source retrieval condition, which was just the opposite of our results and will be discussed below. The last possible factor is the guessing strategy. Different from item recall, guessing could be an important influencing factor in source memory since it required only a forced answer between two choices. Unfortunately, we failed to address this uncontrolled factor, so we strongly recommend future research to reduce guessing through experimental manipulations like adding a response option of "I don't know" during source retrieval.

\section{Beneficial Effects of Error Pruning and False Memory Reduction for Deese-Roediger-McDermott Lists During Collaborative Remembering}

Compared with studies of collaborative inhibition, only a handful of literature focused on the impact of collaboration upon retrieval errors, which revealed that collaborative groups commit fewer errors than nominal groups (Harris et al., 2012; Hyman et al., 2013; Ke et al., 2017). The current study, apart from replicating the standard collaborative inhibition, showed more errors in nominal than collaborative groups, indicating a significant effect of error pruning in DRM lists. Andrews and Rapp (2015) argued that error pruning might be explained partly as people's tendency to adopt a conservative criterion to recall words in a collaborative context out of fear of evaluation from others for making mistakes. The RSDH offers a second possible view - error pruning is the result of error rectification through feedback from collaborative partners (Rajaram, 2011; Rajaram \& Pereira-Pasarin, 2010). However, this was not always the case, since errors of collaborative groups might be influenced by the collaborative style: error pruning tended to be stronger in the case of a free-flowing procedure, which permitted discussion and error correction, but tended to be weaker in a turn-taking procedure, which prohibited participants from talking and correcting errors for each other (Basden et al., 1997; Meade \& Roediger, 2009; Thorley \& Dewhurst, 2007). When comparing the two testing conditions, much larger error pruning was found on source retrieval, which differed from the finding of our recent study (Ke et al., 2017). One possible interpretation might be that it was due to the difference between the nature of the semantically related and unassociated words, but this issue requires further investigation.

Turning to the incorrectly retrieved critical lures, our data revealed that nominal groups produced more false memories than collaborative groups, in accordance with some previous research applying DRM lists (Maki et al., 2008; Saraiva et al., 2017; Weigold et al., 2014). The current benefit of false memory reduction could be explained by the theory of activation/monitoring (Roediger, Balota, \& Watson, 2001). This theory interprets false memories as the result of two processes: activation of the critical lures and failure to monitor the sources for their occurrence. Both paths could contribute to the beneficial effect of false memory reduction during collaborative remembering. Concerning the activation process, the nonpresented critical word would be activated as an implicit response to its semantic associates in the study lists. In the nominal groups, participants could recall more presented words from the same list in comparison with the collaborative groups, which could result in larger activation and enhanced probability of false remembering of the critical lures during retrieval, given the fact that the strength of spreading activation is a function of the total number of activated words (Saraiva et al., 2017).

As for the monitoring process, nominal participants could be more vulnerable to false memories because of their failures to monitor whether the critical lures were generated internally or were externally presented on word lists (i.e., reality monitoring), and because of the lack of partners, whereas the partners in collaborative dyads could serve as monitors for each other to rectify mistakes (Saraiva et al., 2017; Takahashi, 2007). Additional evidence could be gathered for the monitoring account since collaborative groups produced much more false memories versus nominal groups in the turn-taking procedure. That is, no monitoring of retrieval was possible due to the prohibition of discussion. Participants might have also experienced higher pressure from preparing for the upcoming communication during collaboration, thus causing an increase in the false recall of the critical lures (Thorley \& Dewhurst, 2007).

Based on the activation/monitoring hypothesis, the attempt to set the source memory test has further implications regarding false memory. As stated before, the production of a nonstudied critical lure could be attributed to the failure of reality monitoring. False memory could be abated by introducing additional source information that aids source monitoring (Bodner, Huff, Lamontagne, \& Azad, 2017; Huff \& Bodner, 2013; Nieznański, Obidziński, Niedziałkowska, \& Zyskowska, 2018). For instance, front colors were set as additional contextual details in the current study and when participants were required to finish the recall task, the awareness that a critical lure coming to mind as a recall candidate was without color information could aid in rejection, therefore leading to reduced false memory. The collaborative memory paradigm offers an appropriate method to explore whether source monitoring in groups can be a more effective tool than individual monitoring to mitigate the DRM illusion. However, the current study did not include the condition of pure item recall without source retrieval, so we were unable to tell whether the reduction of false memory through source monitoring in the collaborative dyads would overshadow that under the condition where no subsequent source retrieval was demanded.

\section{Two Beneficial Effects for Deese- Roediger-McDermott Lists After Collaborative Remembering}

Turning to Recall 2, there were two beneficial effects. First, our current study replicated an advantageous effect for collaborative remembering on post-collaborative recall of true memory. As stated before, the RSDH seemed to be the best explanation for such a benefit. This hypothesis posits that a subsequent individual retrieval task could enable foregoing collaborative members to be released from prior retrieval strategy disruptions and revert to their own, probably more efficient, retrieval 
strategies. At least three pathways contribute to the generation of the post-collaborative recall benefit during collaboration: re-exposure to events recalled by their partners, relearning already known information through collaborative retrieval, and receiving error correction from collaborative partners (Blumen et al., 2014; Blumen \& Rajaram, 2008; Harris et al., 2012; Marion \& Thorley, 2016; Rajaram \& PereiraPasarin, 2010; Wissman \& Rawson, 2015). Congleton and Rajaram (2011) showed a similar pattern of post-collaborative individual recall in the case of repeated retrieval learning and suggested that the strengthening of one's own idiosyncratic organization of the studied stimuli protected against the retrieval disruption, which then allowed participants to gain re-exposure benefits during collaboration and therefore improved post-collaborative individual recall. Furthermore, our data showed that the correct recalls were higher for both II and CI groups on item recall versus source retrieval.

Aside from the post-collaborative recall benefits on true memory, there was a beneficial effect of false memory reduction during Recall 2. It suggested not only that the beneficial effect of collaboration for false memory persisted through both recall phases, but also that it occurred in the same way both during and after collaboration. However, we did not find a significant beneficial effect on error pruning during Recall 2, which was not the case during Recall 1 . A possible explanation was that falsely recalled critical and noncritical items might be generated and monitored in different ways. Critical lures are the results of activation through semantic relations, while the incorrectly outputted noncritical items are spontaneous with more diversified groundings. For instance, the phonological association could also lead to error intrusions, the influence of which on false memory has already been identified $(\mathrm{Qu}$ \& Ding, 2010). For example, when learning the word cold, the word hold may come into mind through activation in the lexical network. Furthermore, sematic activation might also yield noncritical errors. For instance, when learning winter and snow, one may recollect Christmas, while another may recollect glove. To sum up, such noncritical errors display wider individual variations so that they are more difficult to generalize from one participant to another. During Recall 1 of the current study, errors like these benefited from strategic monitoring of others, just as the critical lures did. However, when the collaborative context no longer existed in Recall 2, participants tended to revert to their personalized errors. By contrast, recall of critical lures was more universal across participants, so that monitoring for it might last longer until the Recall 2 session. This issue requires further verification.

\section{Future Directions}

The current study leaves room for improvement in the following aspects. To begin with, in each block of our experiment, there was only one DRM list, unlike in previous studies that had more lists in one study set (see, e.g., Saraiva et al., 2017; Thorley \& Dewhurst, 2007). It is likely that if participants have to switch across lists during recall, different data patterns would exhibit, thus demanding further exploration. Second, future research could consider comparing the influence of collaborative remembering on different types of false memories. As shown in our results, memory errors for critical lures and noncritical items behaved differently in post-collaborative remembering. However, since the noncritical erroneous information was uncontrolled, we were incapable of offering a firm account for their disparity. Subsequent research could probe into this phenomenon with rigorous experimental designs. For example, researchers could compare the modulation of collaboration on false memories between semantic and phonetic DRM lists, or between commonly shared and individual errors. In addition, a sequential paradigm was applied so that the source retrieval in our study actually relied on the item recall. A more direct and pure comparison between item recall and source memory could be made if the two tests were separated. Finally, we did not have access to the BAS per word in each list to the critical word, but it would be pertinent to analyze the relationship between the BAS of items printed in a certain color and the color attributed by the participants to a critical lure.

\section{CONCLUSION}

To conclude, the results of the current study provide better understanding of the costs as well as benefits of collaborative and post-collaborative remembering in DRM lists, with both item recall and source retrieval examined. We obtained a disadvantageous effect of collaborative inhibition, as well as three beneficial effects: error pruning, false memory reduction, and post-collaborative recall benefit. These results show that collaborators could serve as disrupters as well as monitors during collaboration and that these impacts last even till subsequent individual recall. At least three novel implications are provided based on the current results: (a) DRM lists can produce reliable error pruning, (b) both the cost effect of collaborative inhibition and the beneficial effect of error pruning for DRM lists are sensitive to testing conditions, and (c) the modulation of collaboration on false memory occurs in the same way during and after collaborative remembering.

\section{ACKNOWLEDGEMENTS}

This study was supported by the Projects of Zhejiang Provincial Natural Science Foundation of China (Grant No. LY17C090003), of Humanities and Social Sciences, Ministry of Education, China (Grant No. 17YJA190010), of National Natural Science Foundation of China (Grant no. 31300831), and of the Fundamental Research Funds for the Central Universities. The authors declare that they have no conflict of interest.

\section{REFERENCES}

Andersson, J. (2001). Net effect of memory collaboration: How is collaboration affected by factors such as friendship, gender and age? Scandinavian Journal of Psychology, 42, 367-375. doi:

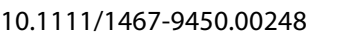

Andrews, J. J., \& Rapp, D. N. (2015). Benefits, costs, and challenges of collaboration for learning and memory. Translational Issues in Psychological Science, 1, 182-191. doi: 10.1037/tps0000025 المبلسليلكا

Barber, S. J., Castrellon, J. J., Opitz, P., \& Mather, M. (2017). Younger and older adults' collaborative recall of shared and unshared emotional pictures. Memory \& Cognition, 45, 1-15. doi: 


\subsection{8/s13421-017-0694-3 سلس}

Barber, S. J., Harris, C. B., \& Rajaram, S. (2015). Why two heads apart are better than two heads together: Multiple mechanisms underlie the collaborative inhibition effect in memory. Journal of Experimental Psychology: Learning, Memory, and Cognition, 41, 559-566. doi: 10.1037/xIm0000037 السلبل

Barber, S. J., Rajaram, S., \& Aron, A. (2010). When two is too many: Collaborative encoding impairs memory. Memory \& Cognition, 38, 255-264. doi: 10.3758/MC.38.3.255 البسلس

Barber, S. J., Rajaram, S., \& Fox, E. B. (2012). Learning and remembering with others: The key role of retrieval in shaping group recall and collective memory. Social Cognition, 30, 121-132. doi: 10.1521/soco.2012.30.1.121 الس السلسلس

Barredo, J., Öztekin, I., \& Badre, D. (2015). Ventral fronto-temporal pathway supporting cognitive control of episodic memory retrieval. Cerebral Cortex, 25, 1004-1019. doi: 10.1093/cercor/

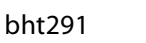

Bärthel, G. A., Wessel, I., Huntjens, R. J., \& Verwoerd, J. (2017). Collaboration enhances later individual memory for emotional material. Memory, 25, 636-646. doi:

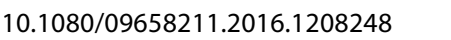

Basden, B. H., Basden, D. R., \& Henry, S. (2000). Costs and benefits of collaborative remembering. Applied Cognitive Psychology, 14, 497-507. doi: 10.1002/1099-0720(200011/12)14:6<497::AIDACP665>3.0.CO;2-4 سلس لس

Basden, B. H., Basden, D. R., Bryner, S., \& Thomas, R. L. (1997). A comparison of group and individual remembering: Does collaboration disrupt retrieval strategies? Journal of Experimental Psychology: Learning, Memory, and Cognition, 23, 1176-1189. doi: 10.1037/0278-7393.23.5.1176 سلاسلس

Basden, D. R., Basden, B. H., \& Stephens, J. P. (2002). Part-set cuing of order information in recall tests. Journal of Memory and Language,

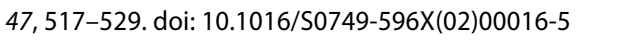

Bell, R., Mieth, L., \& Buchner, A. (2017). Emotional memory: No source memory without old-new recognition. Emotion, 17, 120-130. doi: 10.1037/emo0000211 سلس

Blumen, H. M., \& Rajaram, S. (2008). Influence of re-exposure and retrieval disruption during group collaboration on later individual recall. Memory, 16, 231-244. doi: 10.1080/09658210701804495 الس الس الس الس

Blumen, H. M., \& Stern, Y. (2011). Short-term and long-term collaboration benefits on individual recall in younger and older adults. Memory \& Cognition, 39, 147-154. doi: 10.3758/s13421010-0023-6 سلس

Blumen, H. M., Rajaram, S., \& Henkel, L. (2013). The applied value of collaborative memory research in aging: Behavioral and neural considerations. Journal of Applied Research in Memory and Cognition, 2, 107-117. doi: 10.1016/j.jarmac.2013.03.003 الس السلس

Blumen, H. M., Young, K. E., \& Rajaram, S. (2014). Optimizing group collaboration to improve later retention. Journal of Applied Research in Memory and Cognition, 3, 244-251. doi:

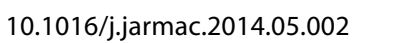

Bodner, G. E., Huff, M. J., Lamontagne, R. W., \& Azad, T. (2017). Getting at the source of distinctive encoding effects in the DRM paradigm: Evidence from signal-detection measures and source judgments. Memory, 25, 647-655. doi:

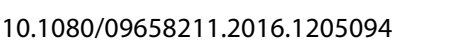

Congleton, A. R., \& Rajaram, S. (2011). The influence of learning methods on collaboration: Prior repeated retrieval enhances retrieval organization, abolishes collaborative inhibition, and promotes post-collaborative memory. Journal of Experimental

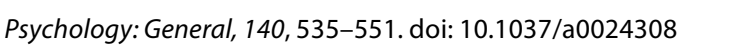

Congleton, A. R., \& Rajaram, S. (2014). Collaboration changes both the content and the structure of memory: Building the architecture of shared representations. Journal of Experimental

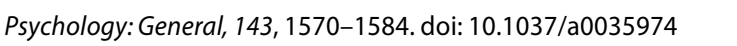
Cooper, E., Greve, A., \& Henson, R. N. (2017). Assumptions behind scoring source versus item memory: Effects of age, hippocampal lesions and mild memory problems. Cortex, 91, 297-315. doi: 10.1016/j.cortex.2017.01.001 1لالسلسلس

Cycowicz, Y. M., \& Friedman, D. (2003). Source memory for the color of pictures: Event-related brain potentials (ERPs) reveal sensory-specific retrieval-related activity. Psychophysiology, 40, 455-464. doi: 10.1111/1469-8986.00047 السلسلسل

Dulas, M. R., \& Duarte, A. (2013). The influence of directed attention at encoding on source memory retrieval in the young and old: An ERP study. Brain Research, 1500, 55-71. doi: 10.1016/j. brainres.2013.01.018

Faul, F., Erdfelder, E., Buchner, A., \& Lang, A. G. (2009). Statistical power analyses using $G^{*}$ Power 3.1: Tests for correlation and regression analyses. Behavior Research Methods, 41, 1149-1160. doi: 10.3758/BRM.41.4.1149 الس الس

Garrido, M. V., Garcia-Marques, L., \& Hamilton, D. L. (2012). Enhancing the comparability between part-list cueing and collaborative recall: A gradual part-list cueing paradigm. Experimental Psychology, 59, 199-205. doi: 10.1027/1618-

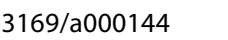

Harris, C. B., Barnier, A. J., \& Sutton, J. (2012). Consensus collaboration enhances group and individual recall accuracy. The Quarterly Journal of Experimental Psychology, 65, 179-194. doi:

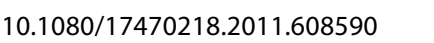

Harris, C. B., Barnier, A. J., \& Sutton, J. (2013). Shared encoding and the costs and benefits of collaborative recall. Journal of Experimental Psychology: Learning, Memory, and Cognition, 39,

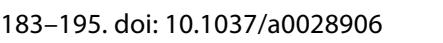

Harris, C. B., Barnier, A. J., Sutton, J., \& Keil, P. G. (2014). Couples as socially distributed cognitive systems: Remembering in everyday social and material contexts. Memory Studies, 7, 285-297. doi: 10.1177/1750698014530619 السلسلس

Harris, C. B., Barnier, A. J., Sutton, J., Keil, P. G., \& Dixon, R. A. (2017). "Going episodic": Collaborative inhibition and facilitation when long-married couples remember together. Memory, 25, 1148-1159. doi: 10.1080/09658211.2016.1274405 سلس

Hayama, H. R., Vilberg, K. L., \& Rugg, M. D. (2012). Overlap 
between the neural correlates of cued recall and source memory: Evidence for a generic recollection network? Journal of Cognitive Neuroscience, 24, 1127-1137. doi 10.1162/ jocn_a_00202 سلس الس الس

Huff, M. J., \& Bodner, G. E. (2013). When does memory monitoring succeed versus fail? Comparing item-specific and relational encoding in the DRM paradigm. Journal of Experimental Psychology: Learning, Memory, and Cognition, 39, 1246-1256. doi: 10.1037/a0031338 سلس

Hyman, I. E., Jr., Cardwell, B. A., \& Roy, R. A. (2013). Multiple causes of collaborative inhibition in memory for categorised word lists. Memory, 21, 875-890. doi: 10.1080/09658211.2013.769058 1ل

Karau, S. J., \& Williams, K. D. (1993). Social loafing: A meta-analytic review and theoretical integration. Journal of Personality and Social Psychology, 65, 681-706. doi: 10.1037/0022-

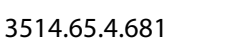

Ke, C., Nie, A., \& Zhang, R. (2017). The modulation of recall task on collaborative inhibition and error pruning: The influence of emotional valence and level of processing. Acta Psychologica Sinica, 49, 733-744. doi: 10.3724/SP.J.1041.2017.00733 السالسالسالس

Kragel, J. E., \& Polyn, S. M. (2016). Decoding episodic retrieval processes: Frontoparietal and medial temporal lobe contributions to free recall. Journal of Cognitive Neuroscience, 28, 125-139. doi: 10.1162/jocn_a_00881 1. Wh

Leynes, P. A., Crawford, J. T., Radebaugh, A. M., \& Taranto, E. (2013). Event-related potential evidence of accessing gender stereotypes to aid source monitoring. Brain Research, 1491, 176-187. doi: 10.1016/j.brainres.2012.11.013 البلسلس

McCabe, D. P., Roediger, H. L., \& Karpicke, J. D. (2011). Automatic processing influences free recall: Converging evidence from the process dissociation procedure and remember-know judgments. Memory \& Cognition, 39, 389-402. doi: 10.3758/

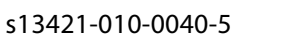

Maki, R., Weigold, A., \& Arrelano, A. (2008). False memory for associated word lists in individuals and collaborating groups. Memory \& Cognition, 36, 598-603. doi: 10.3758/MC.36.3.598 سلس

Malejka, S., \& Bröder, A. (2016). No source memory for unrecognized items when implicit feedback is avoided. Memory \&

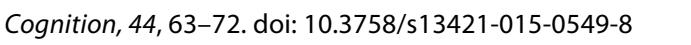

Marion, S. B., \& Thorley, C. (2016). A meta-analytic review of collaborative inhibition and postcollaborative memory: Testing the predictions of the retrieval strategy disruption hypothesis. Psychological Bulletin, 142, 1141-1164. doi: 10.1037/

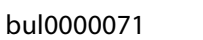

Meade, M. L., \& Roediger, H. L. (2009). Age differences in collaborative memory: The role of retrieval manipulations. Memory \&

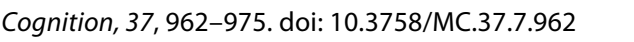

Mitchell, K. J., \& Johnson, M. K. (2009). Source monitoring 15 years later: What have we learned from $\mathrm{fMRI}$ about the neural mechanisms of source memory? Psychological Bulletin, 135, 638-677. doi: 10.1037/a0015849

Mollison, M. V., \& Curran, T. (2012). Familiarity in source memory.
Neuropsychologia, 50, 2546-2565. doi: 10.1016/j.neuropsychologia.2012.06.027 البلالسلسلا

Nie, A., Guo, C., \& Shen, M. (2011). The influence of the testing paradigm on location source retrieval: An event-related potentials study. Acta Psychologica Sinica, 43, 473-482. doi: 10.1016/j.neuropsychologia.2012.06.027 (لالسلسلة

Nie, A., Guo, C., Liang, J., \& Shen, M. (2013). The effect of late posterior negativity in retrieving the color of Chinese characters. Neuroscience Letters, 534, 223-227. doi: 10.1016/j.neulet.2012.11.043 السلسلس

Nie, A., Jiang, J., Fu, Q., \& Zhang, R. (2015). The conjunction effect and feature effect in faces are modulated by task type. Acta Psychologica Sinica, 47, 570-583. doi: 10.3724/ SP.J.1041.2015.00570 سلسلس

Nieznański, M., Obidziński, M., Niedziałkowska, D., \& Zyskowska, E. (2018). Context recollection and false memory of critical lures in the Deese/Roediger-Mcdermott paradigm. Psihologijske Teme, 27, 365-384. Wلس

Numbers, K. T., Meade, M. L., \& Perga, V. A. (2014). The influences of partner accuracy and partner memory ability on social false memories. Memory \& Cognition, 42, 1225-1238. doi: 10.3758/ s13421-014-0443-9 (1) Wل

Qu, Z., \& Ding, Y. (2010). The effect of Chinese phonological associa-

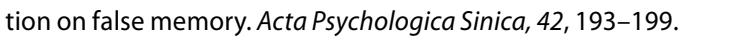

Rajan, V., \& Bell, M. A. (2015). Developmental changes in fact and source recall: Contributions from executive function and brain electrical activity. Developmental Cognitive Neuroscience, 12, 1-11. doi: 10.1016/j.den.2014.10.001 سلسلس

Rajaram, S. (2011). Collaboration both hurts and helps memory: A cognitive perspective. Current Directions in Psychological Science, 20, 76-81. doi: 10.1177/0963721411403251 البلسلسل

Rajaram, S., \& Pereira-Pasarin, L. P. (2010). Collaborative memory: Cognitive research and theory. Perspectives on Psychological Science, 5, 649-663. doi: 10.1177/1745691610388763 الس W.

Roediger, H., \& McDermott, K. (1995). Creating false memories: Remembering words not presented in lists. Journal of Experimental Psychology: Learning, Memory, and Cognition, 21, 803-814. doi: 10.1037/0278-7393.21.4.803 الس السلسلة

Roediger, H., Balota, D., \& Watson, J. (2001). Spreading activation and the arousal of false memories. In H. L. Roediger, J. S. Nairne, I. Neath, \& A. M. Surprenant (Eds.), The nature of remembering: Essays in honor of Robert G. Crowder (pp. 95-115). Washington, DC: American Psychological Association.

Saraiva, M., Albuquerque, P. B., \& Arantes, J. (2017). Production of false memories in collaborative memory tasks using the DRM paradigm. Psicológica, 38, 209-229. Wلسلسلسلس

Sjolund, L. A., Erdman, M., \& Kelly, J. W. (2014). Collaborative inhibition in spatial memory retrieval. Memory \& Cognition, 42,

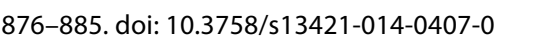

Takahashi, M. (2007). Does collaborative remembering reduce false memories? British Journal of Psychology, 98, 1-13. doi: 10.1348/000712606X101628 سلس 
Thorley, C., \& Dewhurst, S. A. (2007). Collaborative false recall in the DRM procedure: Effects of group size and group pressure. European Journal of Cognitive Psychology, 19, 867-881. doi:

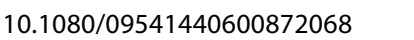

Ventura-Bort, C., Low, A., Wendt, J., Molto, J., Poy, R., Dolcos, F., . . .Weymar, M. (2016). Binding neutral information to emotional contexts: Brain dynamics of long-term recognition memory. Cognitive, Affective \& Behavioral Neuroscience, 16, 234-247. doi: 10.3758/s13415-015-0385-0 السلسلس الس

Weigold, A., Russell, E., \& Natera, S. (2014). Correction of false memory for associated word lists by collaborating groups. American Journal of Psychology, 127, 183-190. doi: 10.5406/ amerjpsyc.127.2.0183 السلسلس

Weldon, M. S., \& Bellinger, K. D. (1997). Collective memory: Collaborative and individual processes in remembering. Journal of Experimental Psychology: Learning, Memory, and Cognition, 23, 1160-1175. doi: 10.1037/0278-7393.23.5.1160 السلسلس

Weldon, M. S., Blair, C., \& Huebsch, P. D. (2000). Group remembering: Does social loafing underlie collaborative inhibition? Journal of Experimental Psychology: Learning, Memory, and Cognition, 26, 1568-1577. doi: 10.1037/0278-7393.26.6.1568 |سلس

Wessel, I., Zandstra, A. R. E., Hengeveld, H. M., \& Moulds, M. L. (2015). Collaborative recall of details of an emotional film.
Memory, 23, 437-444. doi: 10.1080/09658211.2014.895384 سلبل Wissman, K. T., \& Rawson, K. A. (2015). Why does collaborative retrieval improve memory? Enhanced relational and itemspecific processing. Journal of Memory \& Language, 84, 75-87. doi: 10.1016/j.jml.2015.05.003 السلس

Wixted, J. T. (2007). Dual-process theory and signal-detection theory of recognition memory. Psychological Review, 114, 152-176. doi: 10.1037/0033-295X.114.1.152 السلسلس

Yick, Y. Y., \& Wilding, E. L. (2014). Electrophysiological correlates of processes supporting memory for faces. Brain and Cognition,

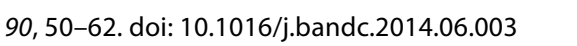

Yonelinas, A. P., Aly, M., Wang, W. C., \& Koen, J. D. (2010). Recollection and familiarity: Examining controversial assumptions and new directions. Hippocampus, 20, 1178-1194. doi: 10.1002/hipo.20864 السلسلس

Yonelinas, A. P., \& Parks, C. M. (2007). Receiver operating characteristics (ROCs) in recognition memory: A review. Psychological Bulletin, 133, 800-832. doi: 10.1037/0033-2909.133.5.800 المالسلس 


\section{APPENDIX: CRITICAL LURES AND WORDS WITHIN EACH LIST, IN ENGLISH}

Needle: thread, pin, eye, sewing, sharp, point, prick, thimble, haystack, thorn, hurt, injection.

Cold: hot, snow, warm, winter, ice, wet, frigid, chilly, weather, freeze, air, shiver. Mountain: hill, valley, climb, top, molehill, peak, plain, glacier, goat, bike, climber, range.

Sleep: bed, rest, awake, tired, dream, wake, snooze, blanket, doze, slumber, snore, nap.

Sweet: sour, candy, sugar, bitter, good, taste, tooth, nice, honey, soda, chocolate, heart.

Chair: table, sit, legs, seat, couch, desk, recliner, sofa, wood, cushion, swivel, stool.

Doctor: nurse, sick, lawyer, medicine, health, hospital, dentist, physician, ill, patient, office, stethoscope. 